\title{
Applying Distortion-Oriented Displays to Groupware
}

\author{
Saul Greenberg and Carl Gutwin \\ Department of Computer Science \\ University of Calgary, Calgary, Canada \\ Tel: +1-403-220-6087 \\ E-mail: saul or gutwin@cpsc.ucalgary.ca
}

\begin{abstract}
This video illustrates three distortion-oriented displays as novel ways of providing awareness of others within groupware. These displays employ magnification lenses and fisheye view techniques to show global context and local detail within a single window, providing both peripheral and detailed awareness of other participants' presence, location, and actions. The head-up lens uses a transparent interface, showing local detail in the foreground and an overview in the background. The offset lens allows a person to magnify a region of the overview. Finally, the fisheye text viewer displays multiple focal points, one for each participant in the conference .
\end{abstract}

\section{INTRODUCTION}

Real-time distributed groupware typically provides a shared virtual workspace where people can see and manipulate work artifacts. The problem is that groupware workspaces do not yet afford the richness of interaction available in their physical counterparts. In particular, it is difficult to maintain a sense of workspace awareness: the up-to-theminute knowledge about another person's interactions with the shared workspace $[4,5]$. One solution provides users with separate awareness windows alongside a conventional full sized viewport. These add-on windows supply either an overview of the entire workspace, or a detailed view of what the other person can see $[3,4,5]$. However, this approach introduces a seam: to gather awareness information, people must attend to and mentally integrate two displays that differ in both scale and physical location.

In this video, we present distortion oriented displays as an alternative mechanism for presenting awareness information [1]. They work by scaling most or all of a workspace to fit within a window, and then distorting (or magnifying) a region to show its detail. When applied to groupware, they provides both peripheral and detailed awareness of other participants by showing their position and actions in the global context, and by distorting the area around their work to see the details of the interaction.

\section{THE HEAD-UP LENS}

The head-up lens (Figure 1) is a transparent user interface with a background and foreground layer [1]. It implements a toy graph editor. The background layer fits a greyed-out

(C) Copyright on this material is held by the authors.

\author{
Andy Cockburn \\ Department of Computer Science \\ University of Canterbury, Christchurch, N.Z. \\ Tel: +64-3-364-2774 \\ E-mail: andy@cosc.canterbury.ac.nz
}

overview of the entire workspace into the window, thus presenting the global context. The see-through foreground shows local detail, which is a person's particular viewport into the workspace. As groupware, editing actions taken by participants are immediately displayed in the overview, giving all an awareness of the changes that are occurring. Uniquely coloured rectangles on the background show the view extents of the local and remote participants, providing location and identity awareness. For example, the foreground viewport in Figure 1 is represented as the midright rectangle in the background. When someone moves their foreground view via their scrollbars, their rectangle slides around the background, showing others where they are currently located. Finally, telepointers (drawn as small circles in the background, and as arrows in the foreground) give some indication of what object others are focusing on.

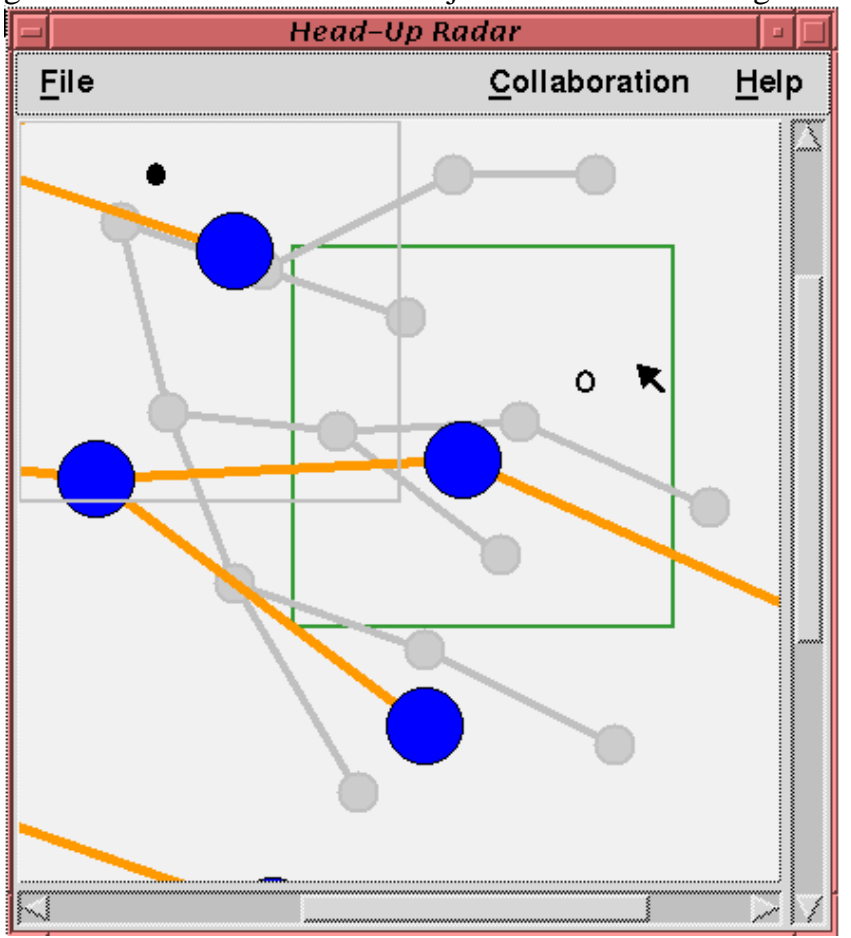

Figure 1: The Head-Up Lens.

\section{THE OFFSET LENS}

The Offset Lens [1] (Figure 2) is a magnification-oriented groupware system whose interface is somewhat similar to the DragMag image magnifier [7]. As with the head-up lens, the workspace is scaled so that the entire graph fits the window. However, the user now has a magnification lens 


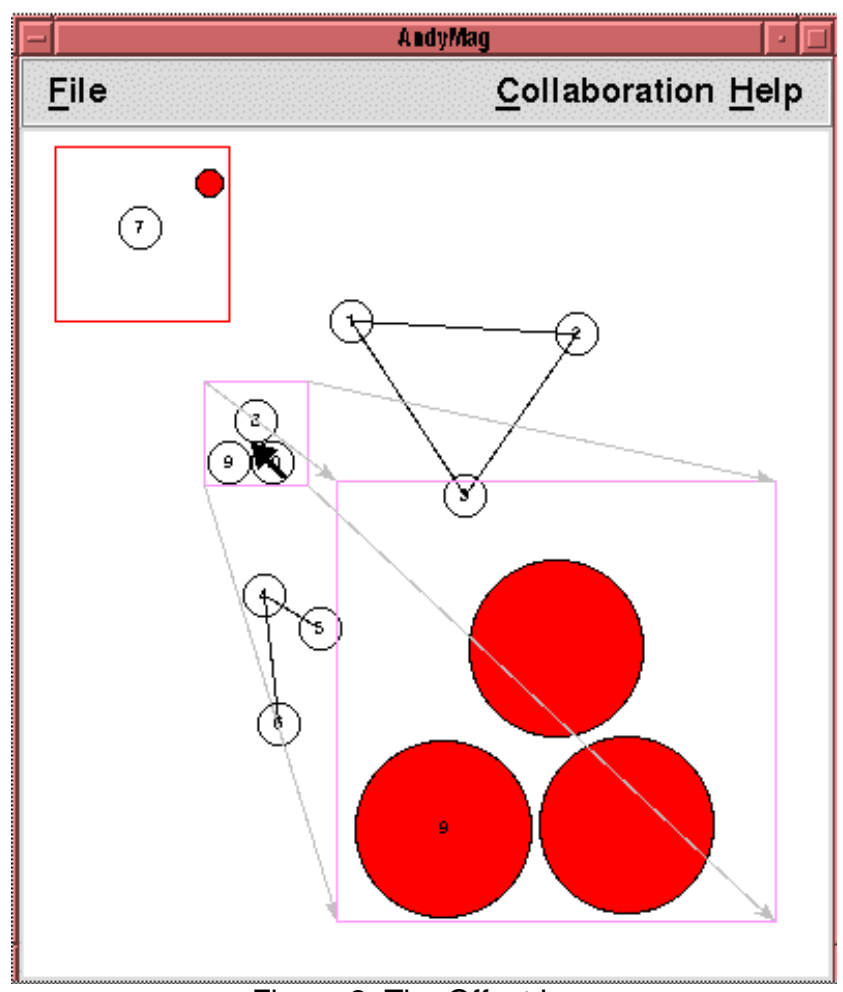

Figure 2: The Offset Lens.

layered over a sub-area of the workspace, shown in Figure 2 as the offset, bordered, and projected area on the bottom right. This lens shows the local detail around a person's cursor: the lens' position on the display follows the cursor as one moves around the global context, and the contents of the local detail continually update to show the new sub-area beneath the lens. Users can edit either the local detail of the global context by shifting modes. As groupware, all users see the same representation of the global context. Participants maintain activity awareness by seeing the immediate updates of other's editing actions on the global context. Each person stays aware of the location of the others by seeing the position of their magnifiers as coloured boxes on the background. Telepointers are also supported for gesturing and location awareness. Finally, people can align their magnified views when sharing of detailed information is required.

\section{THE FISHEYE TEXT VIEWER}

A fisheye view is a visualization technique that provides both local detail and global context in a single contiguous space. For example, Figure 3 uses a fisheye lens to present a text document. Most of the text is shown at a very small font, which gives the person a sense of the document's global structure. The user views local detail by selecting a focal point in the document, and the surrounding text is magnified in place. As groupware, the editor lets multiple people set their own focal point into the document $[1,2]$. Each person's focal point is then displayed as a magnified colored region. Marking others' focal points with an assigned color gives awareness of other's identity and

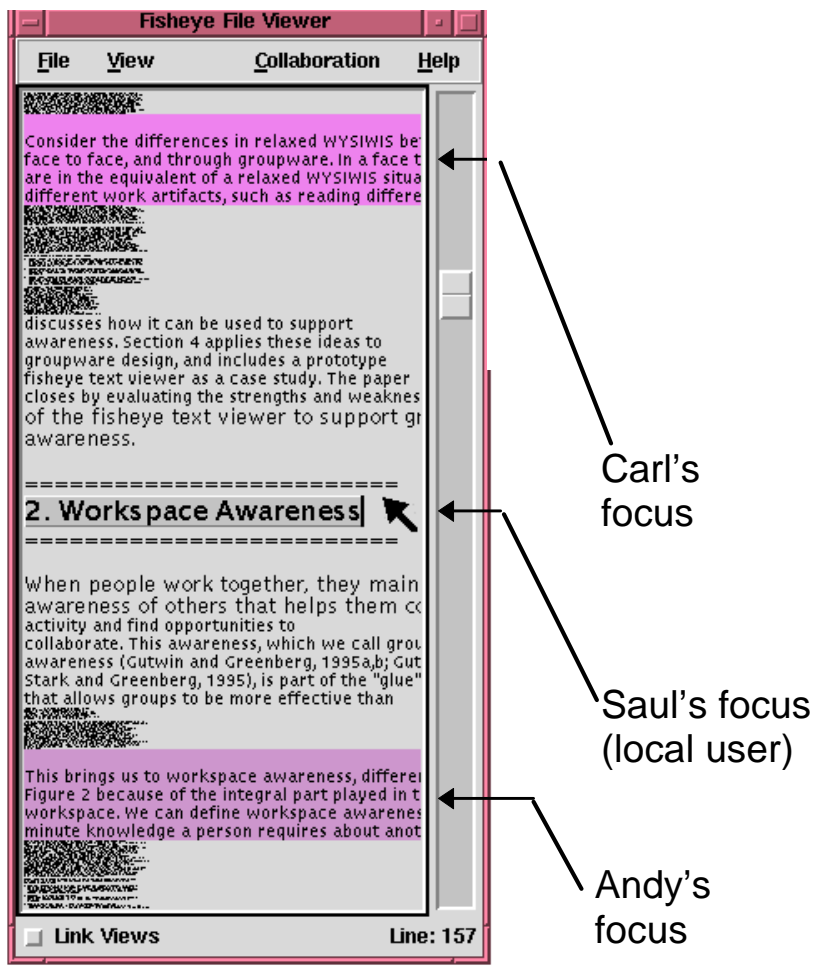

Figure 3: The Fisheye Text Viewer

location. Because the text around other participants' focal points is magnified, as shown in Figure 3, a person can see fine grained details of other's editing actions and how they are situated in the local and global context. For tightlycoupled collaboration, people can link their views, which lets all participants share a common focal point.

\section{REFERENCES}

1. Greenberg, S., Gutwin, C. and Cockburn, A. (1996) Using Distortion-Oriented Displays to Support Workspace Awareness. In [6], p299-314.

2. Greenberg S., Gutwin, C. and Cockburn, A. (1996). Awareness Through Fisheye Views in RelaxedWYSIWIS Groupware. Proceedings Graphics Interface, p28-38. Distributed by Morgan-Kaufmann.

3. Gutwin, C., Greenberg, S., and Roseman, M. (1996). Staying Aware in Groupware Workspaces. CSCW '96 Video Program, ACM Press.

4. Gutwin, C., Greenberg, S. and Roseman, M. (1996). Workspace Awareness in Real-Time Distributed Groupware: Framework, Widgets, and Evaluation. In [6], p281-298.

5. Gutwin, C., Greenberg, S., Roseman, R. (1996) Supporting Awareness of Others in Groupware. ACM SIGCHI'96 Companion Proceedings, p205-215.

6. Sasse, A., Cunningham, R.J. Winder, R. Editors (1996) People and Computers XI, Springer-Verlag.

7. Ware, C. and Lewis, M. (1995). The DragMag Image Magnifier. Chi '95 Video Program. ACM Press. 